\title{
Randomized trials of retosiban versus placebo or atosiban in spontaneous preterm labor
}

George Saade $\mathrm{MD}^{\mathrm{a}}$, Andrew Shennan MD ${ }^{\mathrm{b}}$, Kathleen J Beach MD ${ }^{\mathrm{c}, 1}$, Eran Hadar MD ${ }^{\mathrm{d}}$, Barbara V Parilla $\mathrm{MD}^{\mathrm{e}, 2}$, Jerry Snidow PharmD ${ }^{\mathrm{f}, 3}$, Marcy Powell $\mathrm{MD}^{\mathrm{g}}$, Timothy H Montague $\mathrm{PhD}^{\mathrm{h}}$, Feng Liu $\mathrm{PhD}^{\mathrm{h}, 4}$, Yosuke Komatsu MD ${ }^{\mathrm{i}, 5}$, Laura McKain $\mathrm{MD}^{\mathrm{j}, 6}$, Steven Thornton $\mathrm{DM}^{\mathrm{k}}$ ${ }^{a}$ Department of Obstetrics and Gynecology, University of Texas Medical Branch, Galveston, TX, USA; 'Department of Women and Children’s Health, King’s College London, St Thomas’ Hospital, London, UK; ' Department of Maternal and Fetal Medicine, GSK, Research Triangle Park, NC, USA; ${ }^{\mathrm{d} H e l e n ~ S c h n e i d e r ~ H o s p i t a l ~ f o r ~ W o m e n, ~ R a b i n ~ M e d i c a l ~}$ Center, Petach-Tikva, Israel and Sackler Faculty of Medicine, Tel Aviv University, Tel Aviv, Israel; ${ }^{e}$ Department of Obstetrics and Gynecology, Advocate Lutheran General Hospital, Park Ridge, IL, USA; ${ }^{\mathrm{f}}$ Alternative Discovery and Development, GSK, Research Triangle Park, NC, USA; ${ }^{g}$ Central Safety Department, GSK, Research Triangle Park, NC, USA; ${ }^{\mathrm{h} C l i n i c a l}$ Statistics, Quantitative Sciences, GSK, Collegeville, PA, USA; ${ }^{i}$ Maternal and Neonatal Health Unit, Alternative Discovery \& Development, R\&D, GSK, Research Triangle Park, NC, USA; jPharmacovigilance, PPD, Wilmington, NC, USA; ${ }^{k}$ Barts and The London School of Medicine and Dentistry, Queen Mary University of London, London, UK

${ }^{1}$ At the time of the trial; ${ }^{2}$ At the time of the trial, present address: Rush Center for MaternalFetal Medicine, Aurora, IL, USA; ${ }^{3}$ At the time of the trial; ${ }^{4}$ At the time of the trial, present address: AstraZeneca, Gaithersburg, MD, USA, ${ }^{5}$ At the time of the trial, present address: Clinical Development, Ferring Pharmaceuticals, Parsippany, NJ, USA; ${ }^{6}$ At the time of the trial, present address: Myovant Sciences, Brisbane, CA, USA

Corresponding Author: Professor George Saade, MD 
Department of Obstetrics and Gynecology, The University of Texas Medical Branch, 301 University Blvd, Galveston, TX, 77555, USA

E-mail: gsaade@utmb.edu

Tel: +1 4097470482

Fax: +1 4097725297

Abstract Word Count: 300 (max 300)

Main Text Word Count: 3225

Running head: Retosiban in spontaneous preterm labor 


\section{Abstract (299/300 words)}

OBJECTIVE: To assess the efficacy and safety of retosiban in spontaneous preterm labor.

STUDY DESIGN: Two multicenter, randomized, double-blind trials compared retosiban with placebo and retosiban with atosiban in women with a singleton pregnancy and intact membranes in spontaneous preterm labor at $24-33^{6 / 7}$ weeks' gestation. Coprimary endpoints in the placebo-controlled trial were time to delivery (TTD) or treatment failure (whichever occurred first) and neonatal composite morbidity and mortality. The primary endpoint of the atosiban comparator trial was TTD.

RESULTS: The trials were terminated early because of slow recruitment. The placebocontrolled trial enrolled 23 participants (February 2016-July 2017; 2.6\% of target); the atosiban-comparator trial enrolled 97 (March 2015-August 2017; 29\% of target). Baseline participant characteristics were similar between treatments. In the placebo-controlled trial, mean gestational ages at randomization were 30.8 (retosiban, $n=10$ ) and 30.5 weeks (placebo, $\mathrm{n}=13$ ), and mean times to delivery/treatment failure were 18.9 days (retosiban) and 11.1 days (placebo). Two and four neonates in the retosiban and placebo groups, respectively, had $\geq 1$ component of the neonatal composite endpoint. In the atosiban-comparator trial, mean gestational age at randomization was 31.5 weeks (for both retosiban, $n=47$, and atosiban, n=50), and adjusted mean TTDs were 32.51 days (retosiban) and 33.71 days (atosiban; $P>0.05)$. Adverse events were no more common with retosiban than placebo or atosiban.

CONCLUSION: Despite considerable efforts to conduct two adequate and well-controlled studies in patients with spontaneous preterm labor, both studies were unable to recruit effectively and consequently terminated prematurely. Key factors negatively affecting participation were patient and physician resistance to use of a placebo comparator, lack of investigator consensus on diagnostic criteria and acceptance of protocol procedures, and 
ethics committee decisions. Meaningful cooperation between pharmaceutical companies, regulatory authorities, and the obstetric community is essential for future development of drugs to treat spontaneous preterm labor.

Key words: atosiban, efficacy, oxytocin antagonist, recruitment, retosiban, safety, spontaneous preterm labor, tocolysis 


\section{Introduction}

Successful development and regulatory approval of treatments for preterm labor have been sparse for 4 decades owing to safety/liability concerns of experimental drugs, regulatory requirements to demonstrate neonatal benefit, changes in clinical management of preterm labor, and the complexity of developing protocols that address these issues. ${ }^{1}$ Several areas central to tocolytic drug development include the feasibility of placebo-controlled trials, identifying appropriate study endpoints and time frames, and ensuring collaboration between the Food and Drug Administration (FDA) and other parties. ${ }^{1}$

In the United States (US), there are no FDA-approved tocolytics ${ }^{1,2}$; atosiban has been approved by the European Medicines Agency (EMA). ${ }^{3}$ Globally, indomethacin, betamimetics, calcium channel blockers, and magnesium sulfate are used off-license. ${ }^{4}$ However, these lack convincing evidence for improvement in neonatal outcomes ${ }^{5,6}$ and may have undesirable fetal and maternal side effects. ${ }^{7,8}$

Retosiban, a selective oxytocin receptor antagonist, was associated with a $>1$-week increase in time to delivery (TTD) versus placebo and a significant reduction in preterm birth, with no safety issues, in a phase 2 trial. $^{2}$

Two randomized trials were conducted in women with spontaneous preterm labor (sPTL) to assess the efficacy and safety of retosiban versus placebo or atosiban; however, the trials were terminated early because of slow recruitment. Here we present the key findings of both studies at trial termination (before calculated sample sizes were reached), review key factors that affected participation, and discuss the challenges of conducting tocolytic trials. 


\section{Materials and Methods}

\section{Trial designs}

The placebo-controlled trial was a phase 3, randomized, double-blind, parallel-group, multicenter trial of retosiban versus placebo in participants with sPTL. The atosiban comparator trial was a multicenter, randomized, double-dummy, double-blind, phase 3 trial of retosiban versus atosiban in participants with sPTL.

Eligible participants were randomized (1:1; Supplementary Material 1) to a 48-hour infusion of assigned treatment. Participants who remained undelivered were assessed 48 hours posttreatment, then contacted weekly until delivery. Maternal delivery and hospitalization records were reviewed by the investigator upon delivery. All participants were contacted within 6 weeks of delivery for assessment, and neonatal records from delivery to 28 days after the estimated date of delivery (EDD; 40 weeks' gestation) were reviewed by a neonatologist. Concomitant tocolytic therapy was prohibited. Participants were eligible for the placebocontrolled trial if: no tocolytic treatment had been initiated before consent; a protocolprohibited tocolytic was stopped before randomization; tocolytic treatment was given in a prior sPTL episode (current pregnancy); or they were receiving magnesium sulfate (and all other eligibility criteria were met). Participants were eligible for the atosiban comparator trial if they had not received any tocolytic or had received but not responded to a tocolytic other than atosiban during their current sPTL episode. Participants were excluded if they had contraindications to tocolytic therapy. Full exclusion criteria and stopping rules are described in Supplementary Material 2.

Antenatal corticosteroids (betamethasone or dexamethasone) were given. Magnesium sulfate and intrapartum antibiotic prophylaxis were permitted at the investigator's discretion, following local guidelines. 
There were no a priori rules for trial termination. Termination was at the discretion of the sponsor, with an independent data monitoring committee able to recommend termination following interim analyses.

\section{Trial populations}

Participants were aged 12-45 years (lower age limit based on prior regulatory discussions with the FDA and EMA) with an uncomplicated singleton pregnancy and intact membranes in sPTL, defined as: regular uterine contractions confirmed by tocodynamometry ( $\geq 4$ contractions of $\geq 30$ seconds' duration during 30 minutes), and cervical dilation $\geq 2-\leq 4 \mathrm{~cm}$ by digital examination or, if $<2 \mathrm{~cm}$, cervical change consistent with a 10 -mm decrease in cervical length by transvaginal ultrasound or an absolute increase of $\geq 25 \%$ in effacement by cervical digital examination or a 1 -cm increase in cervical dilation. Participants were at $24^{0 / 7}$ $33^{6 / 7}$ weeks’ gestation, confirmed/determined by known fertilization date (in vitro fertilization/intrauterine insemination) or EDD (ultrasound before $24^{0 / 7}$ weeks’ gestation).

\section{Treatments}

In both trials, participants were stratified by progesterone treatment (established progesterone therapy vs not) and gestational age $\left(24^{0 / 7}-25^{6 / 7}, 26^{0 / 7}-27^{6 / 7}, 28^{0 / 7}-30^{6 / 7}\right.$, or $\left.31^{0 / 7}-33^{6 / 7}\right)$. In the placebo-controlled trial, participants were randomized to intravenous (IV) retosiban infusion over 48 hours or matching placebo. Retosiban was administered as a 6-mg loading dose over 5 minutes followed by a $6-\mathrm{mg} / \mathrm{h}$ infusion for the remainder of the treatment period.

In the atosiban comparator trial, participants were randomized to IV infusion over 48 hours with either retosiban+matching placebo atosiban or atosiban+matching placebo retosiban. Retosiban was administered as described above. Atosiban was administered as a 6.75-mg loading dose over 1 minute followed by an 18-mg/h infusion for 3 hours, after which the infusion rate was reduced to $6 \mathrm{mg} / \mathrm{h}$ for the remainder of the treatment period. Placebo 
infusions in both trials ( $0.9 \%$ sodium chloride) were matched for the loading dose, and subsequent infusion rates were matched for the treatment period.

In both trials, if there was an inadequate response in contraction frequency/intensity or cervical dilation after the first hour of treatment, another 6-mg dose of retosiban (or placebo) was given over 5 minutes, after which retosiban or placebo was given at $12 \mathrm{mg} / \mathrm{h}$.

No additional study treatment doses were permitted in the placebo-controlled trial. In the atosiban comparator trial, additional doses of study treatment could be given, at the investigator's discretion, to participants with recurrent sPTL $\geq 24$ hours after completing the specified treatment regimen and who still met eligibility criteria.

Infusions and matching placebos were prepared by unblinded pharmacists/qualified individuals. Participants and all other personnel were blinded for the study duration.

\section{Ethical approval and data availability}

Trials were conducted in accordance with the International Council for Harmonisation for Good Clinical Practice and country-specific requirements. Study protocols were approved by the ethics committee or institutional review board at each study center, and are available at www.clinicaltrials.gov (NCT02377466 and NCT02292771). An independent data-monitoring committee monitored maternal, fetal, and neonatal unblinded data throughout the trial. An internal GSK safety review team monitored blinded safety data. All participants provided written informed consent.

\section{Objectives and endpoints}

The objectives were to demonstrate superiority of retosiban in prolongation of pregnancy versus placebo and atosiban, and in reducing the risk of neonatal mortality/morbidity versus placebo. 
The placebo-controlled trial had a coprimary endpoint of TTD or treatment failure (use of a rescue tocolytic), whichever occurred first, and an a priori neonatal composite endpoint of mortality and morbidity outcomes up to 28 days after the EDD (Supplementary Material 3). ${ }^{9}$,

${ }^{10}$ Key secondary endpoints included TTD and proportion of births before 37 weeks' gestation.

The primary endpoint of the atosiban comparator trial was TTD. Key secondary endpoints included proportion of births before 37 weeks' gestation, proportion of births at term (37$41^{6 / 7}$ weeks' gestation), and proportion of neonates with any diagnosis from the neonatal morbidity or mortality composite endpoint.

In both trials, safety assessments included maternal, fetal, and neonatal adverse events (AEs), maternal vital signs, fetal heart rate assessments, baseline electrocardiogram, and clinical laboratory tests. Pharmacokinetic assessments included blood, umbilical cord, and breast milk retosiban concentrations. Other endpoints are listed in Supplementary Material 3.

\section{Statistical analyses}

The recruitment target for the placebo-controlled trial was 900 participants to provide $86 \%$ power to detect a $32 \%$ relative risk reduction between retosiban and placebo in neonatal outcomes and an average difference of 5.5 days in TTD or treatment failure, assuming an incidence of $23 \%$ for retosiban and 34\% for placebo, allowing for interim analysis (planned at 150 and 400 deliveries).

Recruitment target for the atosiban comparator trial was 330 participants, to provide $86 \%$ power to detect an average difference of 9.5 days between retosiban and atosiban in TTD. Interim analysis was planned at 130 deliveries. For both trials, calculations were based on simulations using assumptions based on real-world data and a 2-sided testing procedure with a $\sim 10 \%$ dropout rate. 
Data are presented for the intent-to-treat population, comprising all participants who received study drug and their infants.

Because of the early trial termination and resultant small sample size, no statistical analysis was conducted for the placebo-controlled trial. The coprimary endpoints are summarized using descriptive statistics. The prospectively planned analyses are listed in Supplementary Material 4.

The primary endpoint in the atosiban comparator trial was analyzed using a 2-component normal mixture model. Within each component, expected TTD was modeled as a function of treatment as a fixed effect, and gestational age at randomization and established progesterone use as covariates. Point estimates of the treatment means and standard deviation (SD) were calculated, along with the estimate of mean treatment difference (retosiban-atosiban), corresponding 95\% confidence intervals (CIs), and $P$-values. Time to delivery by treatment was presented in a Kaplan-Meier plot. Key secondary endpoints (Supplementary Material 4) were not analyzed.

All analyses were performed using SAS version 9.3 (SAS Institute Inc., Cary, NC, USA).

\section{Results}

Study sites, participant disposition, and characteristics

Of 607 centers approached for the placebo-controlled trial, 46 centers in 5 countries agreed to recruit (Supplementary Material 5) and 10 centers enrolled participants between February 2016 and July 2017. Screening failures were high: 1\% of participants met eligibility criteria. Twenty-five participants were randomized, and 23 received treatment (10 retosiban, 13 placebo; Figure 1).

Of 343 centers approached in the atosiban comparator trial, 48 centers in 10 countries agreed to participate (Supplementary Material 5), with 23 centers enrolling participants between 
March 2015 and August 2017. Screening failure rates were high: 6\% of participants met eligibility criteria. One hundred participants were randomized and 97 received treatment (47 retosiban, 50 atosiban; Figure 2).

There was no overlap in the centers enrolling for each study (Supplementary Material 5).

In both trials, baseline maternal and fetal demographics and clinical characteristics were similar between treatment groups (Tables 1 and 2).

\section{Treatment}

In the placebo-controlled trial, 3 (30.0\%) participants in the retosiban group required a dose increase versus 10 (76.9\%) in the placebo group. Three (30.0\%) participants in the retosiban group and 5 (38.5\%) in the placebo group discontinued treatment. This was because of an inadequate response in contraction frequency/intensity or cervical dilation in 2 retosibantreated and 4 placebo-treated participants.

In the atosiban comparator trial, 17 (36.2\%) retosiban-treated participants versus 23 (46.0\%) atosiban-treated participants received an increased dose. Eight participants in each group discontinued treatment; 5 in each group owing to an inadequate therapeutic response, 3 to AEs (retosiban: $\mathrm{n}=1$ maternal, $\mathrm{n}=1$ fetal; atosiban: $\mathrm{n}=1$ maternal), and 1 each owing to investigator decision (atosiban), participant decision (atosiban), and other reasons (not stated; retosiban).

\section{Efficacy}

In the placebo-controlled trial, the mean (SD) TTD or treatment failure for retosiban-treated participants was 18.9 (22.99) versus 11.1 (14.99) days for placebo. One retosiban-treated participant met criteria for treatment failure (received the rescue tocolytic ketorolac trometamol). In the placebo group, 4 participants met criteria for treatment failure; of these, 2 received magnesium sulfate at a rate $>2 \mathrm{~g} / \mathrm{h}$ and 2 received rescue tocolytics (terbutaline and 
nifedipine). Two (20.0\%) neonates in the retosiban group and 4 (30.8\%) in the placebo group had $\geq 1$ component of the neonatal composite endpoint. In the retosiban group, both infants experienced respiratory distress syndrome (RDS). In the placebo group, there was 1 infant with RDS, 1 with bronchopulmonary dysplasia, and 2 with RDS and bronchopulmonary dysplasia.

In the placebo-controlled trial, 8 (80.0\%) participants in the retosiban group and $9(69.2 \%)$ in the placebo group delivered before 37 weeks' gestation; 2 (20.0\%) and 4 (30.8\%), respectively, delivered at term. The mean (SD) TTD was 19.2 (22.77) days for retosibantreated participants versus 16.3 (18.60) days with placebo.

In the atosiban comparator trial, the adjusted mean (SD) TTD was 32.5 (2.99) days in the retosiban group and 33.7 (2.53) days in the atosiban group $(P>0.05)$, with a treatment difference of -1.20 days (95\% CI $-8.879,6.479 ; P>0.05$ ) (Figure 3). The mean (SD) times from first study treatment dose until delivery, retreatment, or subsequent preterm labor, whichever occurred first, were 21.7 (18.55) days (retosiban, n=46) and 24.6 (22.06) days (atosiban, n=50). Overall, 25 (54.3\%) participants and 28 (56.0\%) participants receiving retosiban and atosiban, respectively, delivered before 37 weeks' gestation. Three (6.4\%) neonates in the retosiban group and $2(4.0 \%)$ in the atosiban group had a diagnosis within the composite efficacy endpoint (retosiban, RDS n=3; atosiban, RDS n=1, died n=1).

\section{Safety}

Maternal, fetal, and neonatal AEs are listed in Supplementary Material 6.

In the placebo-controlled trial, 6 maternal participants in each treatment group (retosiban 60.0\%; placebo 46.2\%) experienced an $\mathrm{AE}$ (none serious). There were 5 (50.0\%) fetal AEs in the retosiban group, 1 of which was serious but likely unrelated to study treatment (umbilical cord prolapse), and 3 (23.1\%) in the placebo group (none serious). Seven (70.0\%) neonates in 
the retosiban group and 8 (61.5\%) in the placebo group had an AE; 5 (50.0\%) and 3 (23.1\%) were considered serious, respectively. Two neonates, 1 per treatment group, experienced neonatal RDS-related events not included in the composite endpoint (retosiban: did not meet definition of RDS; placebo: captured as an AE but not evaluated for the composite endpoint). No AEs led to treatment discontinuation or study withdrawal or were considered study drug related. There were no deaths.

In the atosiban comparator trial, maternal AEs were more frequent with retosiban $(n=34$ [72.3\%]) than atosiban $(n=28$ [56.0\%]), whereas the frequencies of serious AEs were similar between groups (retosiban: $n=7$ [14.9\%]; atosiban: $n=9$ [18.0\%]). Postpartum hemorrhage was less common with retosiban $(n=1[2.1 \%])$ than atosiban $(n=5[10.0 \%])$. Three participants discontinued treatment because of AEs (retosiban: $n=1$ maternal [appendicitis], $\mathrm{n}=1$ fetal [oligohydramnios]; atosiban: $\mathrm{n}=1$ maternal [abdominal pain]). Fetal AE incidences were similar in each group (retosiban $n=9$ [19.1\%], atosiban $n=8$ [16.0\%]) and 6 were serious (retosiban $n=4$, atosiban $n=2$ ). The incidences of neonatal AEs (retosiban: 25 [53.2\%]; atosiban: 26 [52.0\%]) and serious AEs (retosiban: 10 [21.3\%]; atosiban: 11 [22.0\%]) were similar between groups. Two infants in the retosiban group and 1 in the atosiban group experienced RDS-related events not included in the composite endpoint. No fetal or neonatal AEs were considered to be study drug related. Three retosiban-treated and 2 atosiban-treated maternal participants experienced AEs considered study drug related.

There were no maternal or fetal deaths. There was 1 neonatal death (sudden infant death syndrome) in the atosiban group, which occurred 39 days after delivery.

In both trials, there were no clinically important changes in hematologic or biochemical parameters or maternal vital signs, all of which were similar between treatment groups across visits. 


\section{Pharmacokinetics}

The results of pharmacokinetic analyses on a subset of participants receiving retosiban in the placebo-controlled trial $(n=10)$ and atosiban comparator trial $(n=47)$ are described in Supplementary Material 7.

\section{Comment}

\section{Principal findings and results}

Due to recruitment challenges, both interventional studies were terminated early. For both studies, no new safety signals for retosiban were identified for maternal, fetal, or neonatal participants. As the placebo-controlled study completed with $2.6 \%$ of the planned enrollment, no formal hypothesis testing was conducted on efficacy endpoints, thereby limiting conclusions regarding the effectiveness of retosiban compared with placebo. The atosiban controlled study completed with $29 \%$ of the planned enrollment, and formal hypothesis testing demonstrated no statistically significant difference between the atosiban and retosiban groups to prolong pregnancy. Since there was no overlap between the centers recruiting for each study, and indeed limited overlap in countries involved in each study (Supplementary Material 5), we conclude that conducting two studies concurrently was not a factor in low participant recruitment. Our efforts to increase recruitment included addition of study sites, increasing staff at study sites, and reduction of a requirement for 48-hour continuous fetal heart rate monitoring to a minimum of 6 hours if heart rate remained reassuring.

\section{Clinical and research implications}

Because of early study termination, the clinical implications are limited, apart from the fact that retosiban will not be licensed. Clinical practice in the US will continue to use tocolytics that are not FDA approved and have limited to no evidence of neonatal benefit. However, these studies have broad research implications for future research in this area. 
The FDA requires placebo-controlled trials that are designed and powered to demonstrate neonatal benefit and in which participants receive no other tocolytics. ${ }^{1,11}$ The inclusion of a placebo arm led to many countries and sites refusing to participate as they considered it below standard of care (SoC), even with clinical equipoise. ${ }^{1,11}$ Several alternative trial designs were proposed to the FDA, including placebo in addition to SoC or versus an active comparator (eg, nifedipine). Meetings were held with the FDA, which included key opinion leaders in maternal fetal medicine and leaders on clinical trial design, and a clear path was provided for how the analysis would be managed to account for the treatment variables. These proposals were rejected by the FDA despite precedented use in many therapeutic areas. Conversely, add-on and active-control study designs were acceptable to the EMA.

The low frequency of individual endpoints requires large sample sizes. ${ }^{1}$ The planned placebocontrolled trial would have taken $>10$ years to complete. In the retosiban trials, the sample size required to show a meaningful treatment difference in the neonatal composite endpoint was a major barrier and led to early study termination. A solution in future trials would be to use a composite endpoint, but no standard exists for neonatal morbidity and mortality, ${ }^{1}$ nor are there standardized definitions for some composite endpoint components (eg, RDS), and no regulatory-accepted surrogate endpoint exists. ${ }^{1}$ Neonatology expertise was utilized when developing the composite endpoint for neonatal morbidity used in this study, and background rates were estimated using several different databases and literature. There is a need for the obstetrics community to develop suitable standardized endpoints.

These studies also highlight the challenge of accommodating different treatment practices and SoC between centers, which made it difficult to design a protocol suitable for all centers and acceptable to regulatory agencies. The regulatory agencies required retosiban to be trialed in adolescents; however, in 6 countries, ethics committees did not allow this. French 
investigators did not participate because of the requirement of continuous fetal heart rate monitoring during study drug administration. ${ }^{12}$

The trials were also complicated by lack of consensus on the definition of sPTL, and differing methods used to measure cervical dilation and change. Until differences in diagnostic practices between centers and the lack of defined SoC are resolved, these issues will continue to impact tocolytic development.

\section{Strengths and limitations}

The planned placebo-controlled trial would have represented the largest tocolytic study ever conducted and would have been one of the few studies to compare a tocolytic against placebo. However, the feasibility estimates did not match actual enrollment of participants. One limitation was that the complex protocols introduced challenges for participating sites. Other limitations were related to regulatory requirements for the study design and have been discussed above.

\section{Conclusions}

These trials demonstrate the multifactorial difficulties encountered in designing and conducting clinical trials in sPTL, including a lack of global medical consensus for obstetric and neonatal definitions and care, differing regulatory requirements between the US and elsewhere, and the lack of a validated composite endpoint for neonatal outcomes. Significant cooperation between pharmaceutical companies, regulatory authorities, and the obstetric community is required to standardize criteria to assess future tocolytic agents and overcome the hurdles inherent to trials in pregnant women. Although a simpler way forward would be to conduct regional studies, the number of neonatal events required to show statistical success makes this unfeasible; the FDA has rejected the use of a meta-analysis approach, which could address this limitation. 
In 2006, Goodwin reviewed the challenges in tocolytic development and asked whether a true placebo-controlled trial can be conducted in a timely fashion. ${ }^{1}$ The retosiban trials suggest that no satisfactory solutions have been found. 


\section{Conflicts of Interest}

G.S., E.H., and B.V.P. report no conflicts of interest. A.S. provides consultancy advice for commercial organizations. He is chief investigator on a number of trials funded by the National Institute for Health Research (NIHR; UK) related to the prevention of early birth and improved neonatal outcomes. He has no conflict related to the subject matter of this paper. S.T. provides consultancy advice for commercial organizations such as GSK, Ferring and Hologic. He is a Trustee of a charity that funds related research. He holds positions in the Royal College of Obstetricians and Gynaecologists and other organizations. M.P. and T.H.M. are employees and shareholders of GSK. K.J.B., J.S., F.L., and Y.K. were employees of GSK at the time of the study and are shareholders of GSK. L.M. was an employee of PPD at the time of the study.

\section{Acknowledgments}

We would like to thank the staff at the study sites for their time and contributions to these studies. The authors would like to especially acknowledge Professor Michael O’Shea, University of North Carolina at Chapel Hill, NC, USA (in the past 3 years, has received honoraria from the University of Miami, Duke University, and East Carolina University for visiting professorships; compensation from the American Board of Pediatrics for assistance with developing competency assessment tools for physicians; compensation from the NIH for assistance with grant review and from the University of San Francisco for contributions to a research study on erythropoietin to reduce brain damage in full term infants with neonatal encephalopathy; research funding from the NIH) and Professor Ricki Goldstein, University of Kentucky/Kentucky Children’s Hospital, Lexington, KY, USA (no relevant conflicts of interest to declare) for their expertise in neonatology and contributions to the design of the trials. We also acknowledge contributors from the retosiban advisory board, including Dr Steve N. Caritis, University of Pittsburgh, Pittsburgh, PA, USA (no relevant conflicts of 
interest to declare); Professor Gian Carlo Di Renzo, Department of Obstetrics and Gynecology, University of Perugia, Perugia, Italy (advisor to Ferring Pharmaceuticals); Dr Hugh Miller, Watching Over Mothers and Babies Foundation, AZ, USA (has received research funding from GSK and participated in advisory panels for GSK); Dr Luis SanchezRamos, University of Florida College of Medicine, Jacksonville, FL, USA (no relevant conflicts of interest to declare); Dr Luis Cabero Roura, Hospital Valle Hebron de Barcelona, Barcelona, Spain (no relevant conflicts of interest to declare); Dr Guillermo Valenzuela, Arrowhead Regional Medical Center, CA, USA (also member of the steering committee; no relevant conflicts of interest to declare); and Dr Michael Varner, University of Utah, UT, USA (principal investigator on clinical studies for Novavax and Mellitus, LLC; and has received speaker's fees from Rush University Medical Center, IL, USA, and research funding from the NIH); and the retosiban steering committee, including Dr Gerard HA Visser, Wilhelmina Children’s Hospital, Utrecht, the Netherlands (has acted as a consultant to GSK) and Dr John Van den Anker, Children’s National Health System, Washington, DC, USA (no relevant conflicts of interest to declare). The trials were funded by GSK. GSK contributed to the design of the trials and were involved in the collection, analysis and interpretation of the data and writing of this manuscript. Pharmaceutical Product Development (PPD;

Wilmington, NC, USA) was responsible for safety monitoring and pharmacovigilance, clinical site management, randomization, clinical supplies, data management, regulatory submissions, biostatistics and programming. Medical writing assistance was provided by Katie White, PhD, of Fishawack Indicia Ltd, UK, and was funded by GSK.

\section{Data Sharing Statement}

Within 6 months of this publication, anonymized individual participant data, the annotated case report form, protocol, reporting and analysis plan, data set specifications, raw dataset, analysis-ready dataset, and clinical study report will be available for research proposals 
approved by an independent review committee. Proposals should be submitted to www.clinicalstudydatarequest.com. A data access agreement will be required. 


\section{References}

1. Goodwin TM. Regulatory and methodologic challenges to tocolytic development. BJOG 2006;113:100-04

2. Thornton S, Miller H, Valenzuela G, et al. Treatment of spontaneous preterm labour with retosiban: a phase 2 proof-of-concept study. Br J Clin Pharmacol 2015;80:740 49

3. European Medicines Agency. Tractocile, atosiban. Document EMA/777733/2009. Available at: http://www.ema.europa.eu/docs/en_GB/document_library/EPAR__Summary_for_the_public/human/000253/WC500040489.pdf. Accessed December 20, 2018

4. Royal College of Obstetricians and Gynaecologists. Tocolytic drugs for women in preterm labour. Green-top Guideline No 1B. Available at: http://www.rcog.org.uk/womens-health/clinical-guidance/tocolytic-drugs-womenpreterm-labour-green-top-1b. Accessed December 202018

5. Haas DM, Caldwell DM, Kirkpatrick P, Mcintosh JJ, Welton NJ. Tocolytic therapy for preterm delivery: systematic review and network meta-analysis. The BMJ 2012;345:e6226

6. World Health Organization. WHO recommendations on interventions to improve preterm birth outcomes. Available at: http://www.who.int/reproductivehealth/publications/maternal_perinatal_health/preter m-birth-guideline/en/. Accessed December 20, 2018

7. De Heus R, Mol BW, Erwich JJ, et al. Adverse drug reactions to tocolytic treatment for preterm labour: prospective cohort study. BMJ 2009;338:b744 
8. American College of Obstetricians and Gynecologists. Management of Preterm Labor. Practice Bulletin Number 171. Available at: http://www.acog.org/ResourcesAnd-Publications/Practice-Bulletins-List. Accessed December 20, 2018

9. Pimenta JM, Ebeling M, Montague TH, et al. A retrospective database analysis of neonatal morbidities to evaluate a composite endpoint for use in preterm labor clinical trials. AJP Reports 2018;8:e25-e32

10. Pimenta JM, Brinkley E, Montague TH, Beach KJ, Mack C. Assessing a composite end point for new tocolytics in clinical trials: Data from 4 US integrated delivery networks. Pharmacoepidemiol Drug Saf 2018;27:213-20

11. Goodwin T. The Gordian knot of developing tocolytics. J Soc Gynecol Investig 2004;11:339-41

12. Komatsu Y, Mckain L, Powell M. Prevention of spontaneous preterm birth: Guidelines for clinical practice from the French College of Gynaecologists and Obstetricians (CNGOF). Eur J Obstet Gynecol Reprod Biol 2017;219:130 


\section{Figure Captions}

FIGURE 1 Participant disposition in the placebo-controlled trial

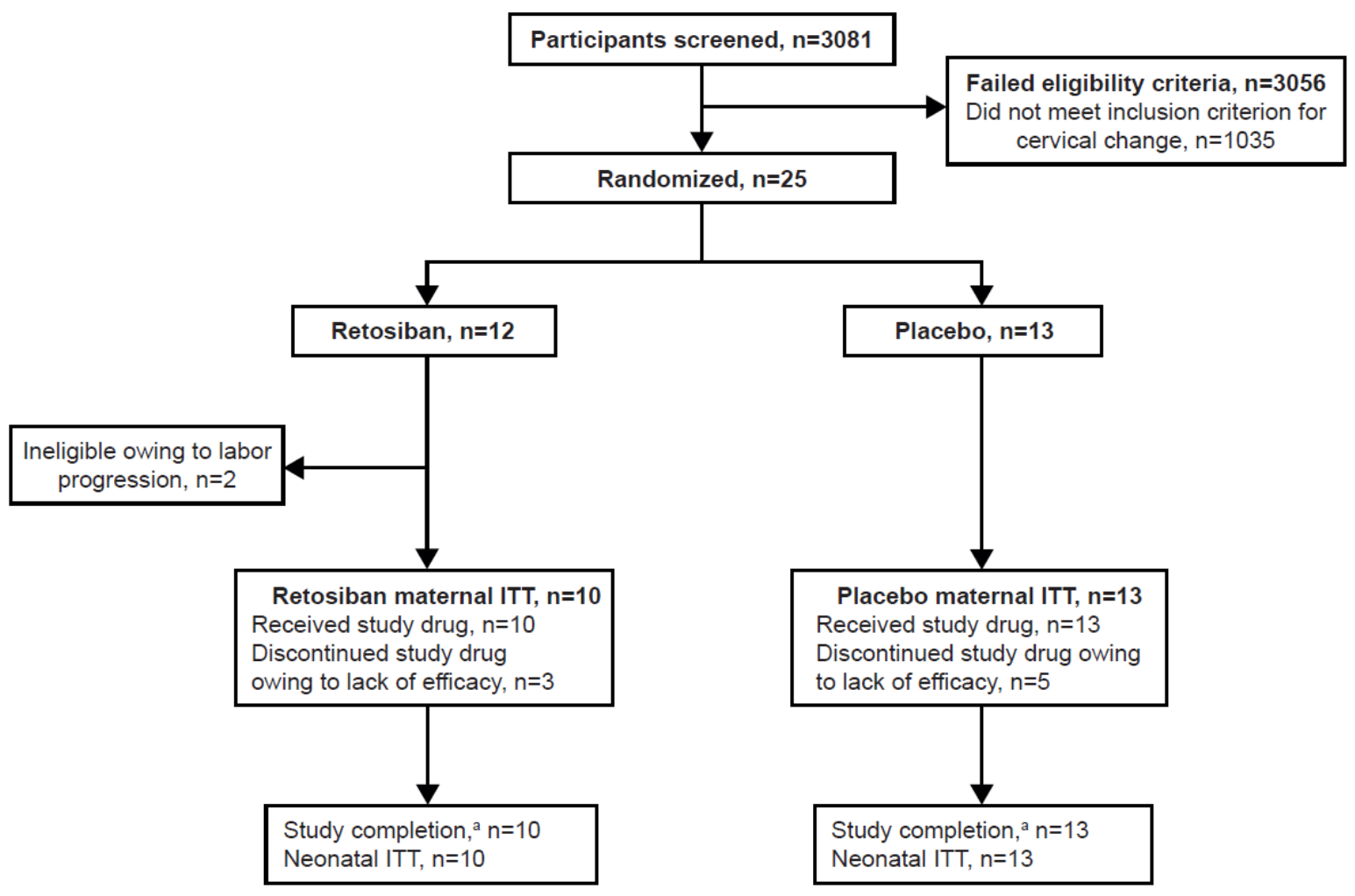

ITT, intent-to-treat

a Defined as completing all stages of the study, including the post-delivery assessment

6 weeks after delivery and the neonatal medical review 28 days after estimated date

of delivery. 
FIGURE 2 Participant disposition in the atosiban comparator trial

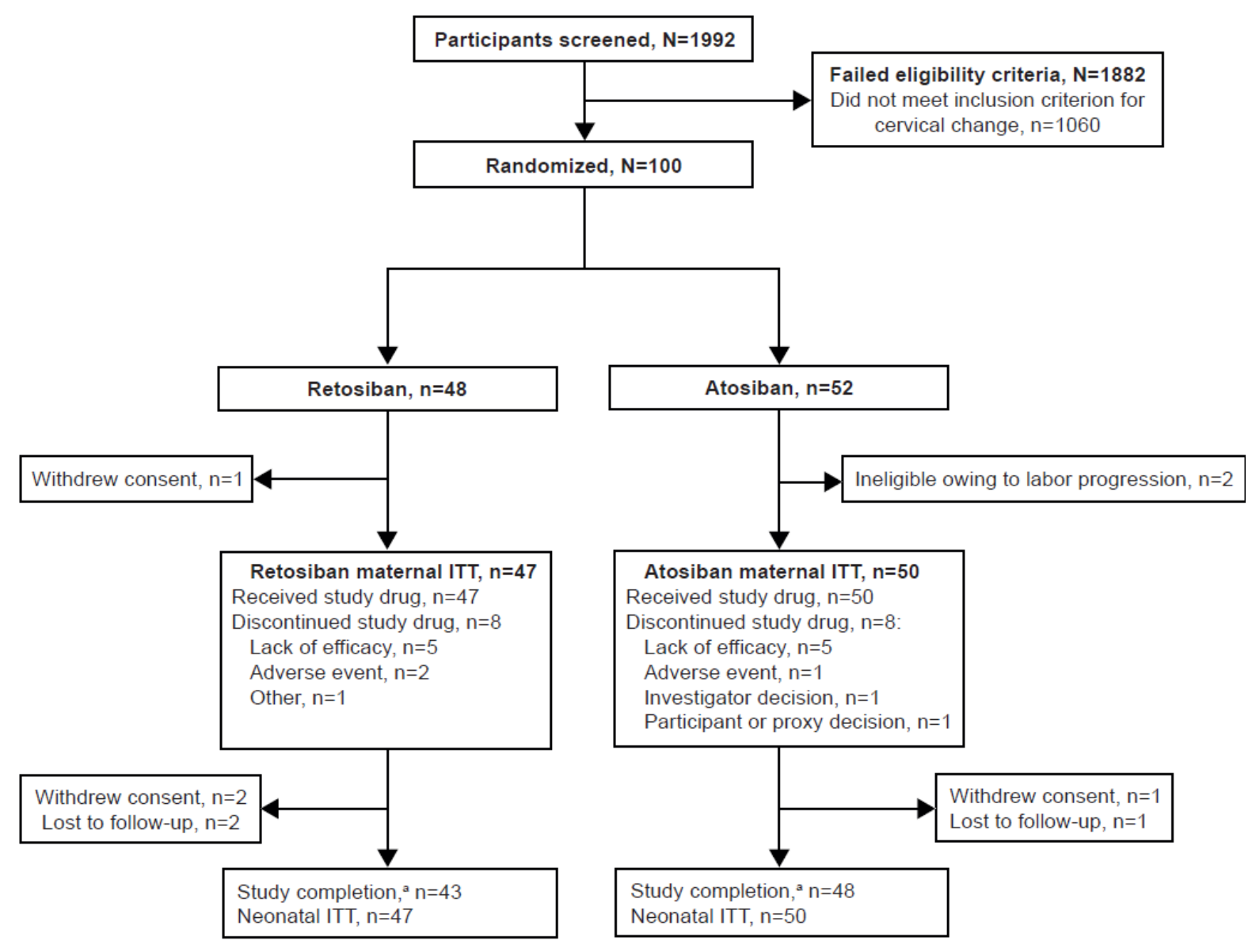

ITT, intent-to-treat

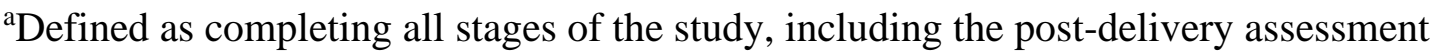

6 weeks after delivery and the neonatal medical review 28 days after estimated date of delivery. 
FIGURE 3 Kaplan-Meier curves of time to delivery in the atosiban comparator trial

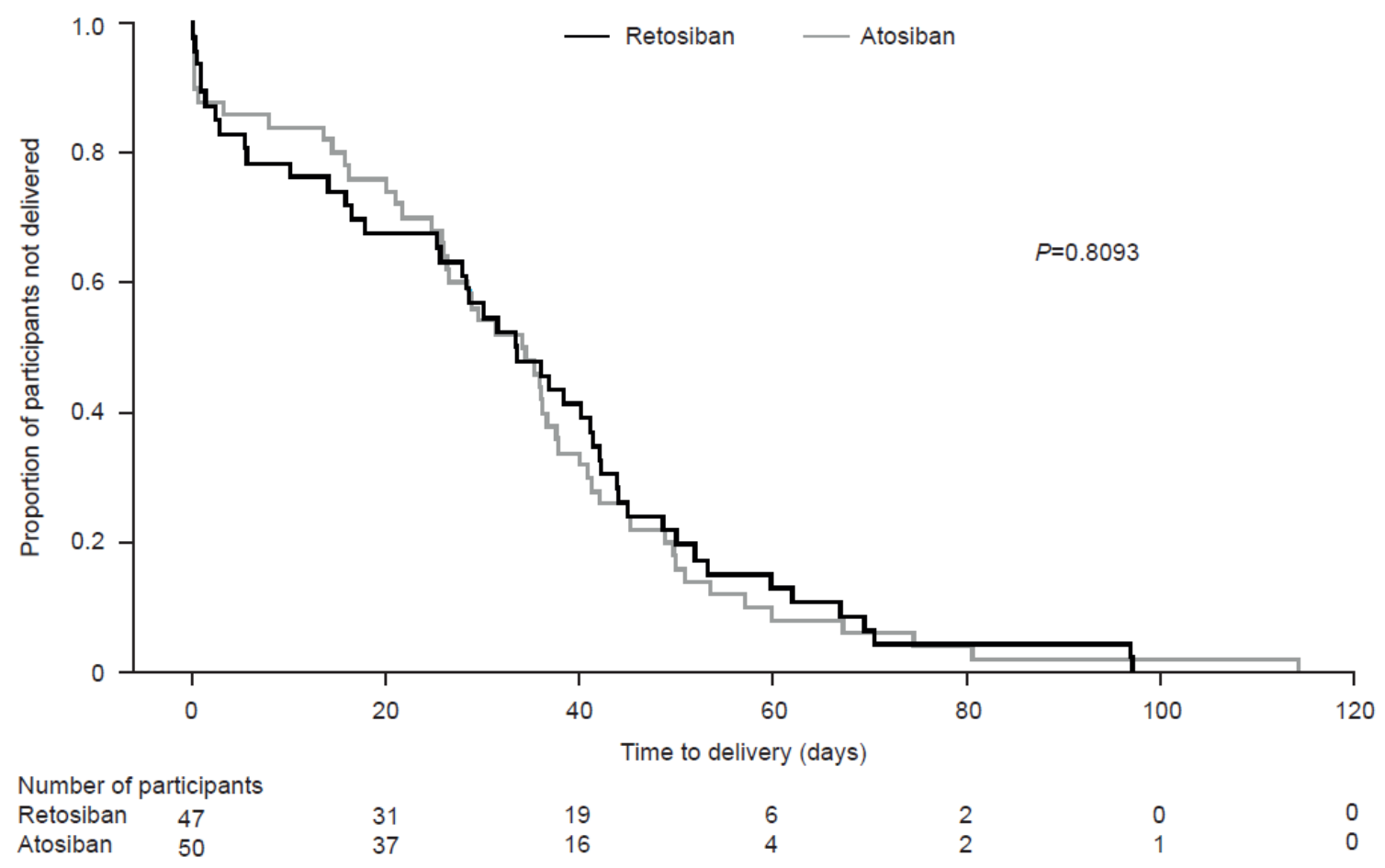

One participant in the retosiban group was withdrawn before delivery and was considered censored at the last record available in the study database; $P$-value is retosiban versus atosiban, based on log-rank test. 


\section{Tables}

TABLE 1 Maternal/fetal baseline demographics and clinical characteristics

\begin{tabular}{|c|c|c|c|c|}
\hline Characteristic & $\begin{array}{l}\text { Retosiban } \\
(\mathrm{N}=10)\end{array}$ & $\begin{array}{l}\text { Placebo } \\
(\mathrm{N}=13)\end{array}$ & $\begin{array}{l}\text { Atosiban co } \\
\text { trial } \\
\text { Retosiban } \\
(\mathrm{N}=47)\end{array}$ & $\begin{array}{l}\text { aparator } \\
\text { Atosiban } \\
(\mathrm{N}=50)\end{array}$ \\
\hline Age, mean $\pm S D, y$ & $27.7 \pm 6.73$ & $26.5 \pm 6.78$ & $27.7 \pm 6.15$ & $27.1 \pm 5.66$ \\
\hline Minimum, maximum & 17,38 & 18,37 & 17,40 & 16,39 \\
\hline \multicolumn{5}{|l|}{ Race, n (\%) } \\
\hline African American/African & $2(20.0)$ & $2(15.4)$ & 0 & $3(6.0)$ \\
\hline American Indian or Alaskan native & 0 & 0 & 7 (14.9) & $4(8.0)$ \\
\hline Asian & $3(30.0)$ & $6(46.2)$ & $9(19.1)$ & $4(8.0)$ \\
\hline White & $5(50.0)$ & $5(38.5)$ & $31(66.0)$ & $39(78.0)$ \\
\hline Body mass index, mean $\pm \mathrm{SD}, \mathrm{kg} / \mathrm{m}^{2}$ & $26.0 \pm 3.60$ & $24.8 \pm 4.72$ & $25.6 \pm 4.07^{\mathrm{a}}$ & $26.4 \pm 5.02^{b}$ \\
\hline GA at randomization, mean \pm SD, week & $30.8 \pm 2.63$ & $30.5 \pm 2.49$ & $31.5 \pm 2.13$ & $31.5 \pm 2.22$ \\
\hline \multicolumn{5}{|l|}{ GA at randomization, $\mathrm{n}(\%)$} \\
\hline $24^{0 / 7}$ to $25^{6 / 7}$ weeks & 0 & $1(7.7)$ & $2(4.3)$ & $2(4.0)$ \\
\hline $26^{0 / 7}$ to $27^{6 / 7}$ weeks & $2(20.0)$ & $1(7.7)$ & 0 & $1(2.0)$ \\
\hline $28^{0 / 7}$ to $30^{6 / 7}$ weeks & $3(30.0)$ & $4(30.8)$ & $13(27.7)$ & $12(24.0)$ \\
\hline $31^{0 / 7}$ to $33^{6 / 7}$ weeks & $5(50.0)$ & 7 (53.8) & $32(68.1)$ & $35(70.0)$ \\
\hline
\end{tabular}




\begin{tabular}{|l|ll|ll|}
\hline Cervical dilation, median (range), cm & 2.0 & 2.0 & $1.50^{\mathrm{a}}$ & 1.00 \\
& $(2.0-4.0)$ & $(2.0-4.0)$ & $(0-4.0)$ & $(0-4.0)$ \\
\hline Contractions per 30 minutes, median (range), & $6(4-9)$ & $7(4-17)$ & $7(3-20)$ & $7(4-16)$ \\
\hline
\end{tabular}

${ }^{a} n=46 ;{ }^{b} n=49$

$G A$, gestational age; $S D$, standard deviation 
TABLE 2 Neonatal birth record

\begin{tabular}{|c|c|c|c|c|}
\hline \multirow[t]{2}{*}{ Characteristic } & \multicolumn{2}{|c|}{ Placebo-controlled trial } & \multicolumn{2}{|c|}{ Atosiban comparator trial } \\
\hline & $\begin{array}{l}\text { Retosiban } \\
(\mathrm{N}=10)\end{array}$ & $\begin{array}{l}\text { Placebo } \\
(\mathrm{N}=13)\end{array}$ & $\begin{array}{l}\text { Retosiban } \\
(\mathrm{N}=47)\end{array}$ & $\begin{array}{l}\text { Atosiban } \\
(\mathrm{N}=50)\end{array}$ \\
\hline $\begin{array}{l}\text { GA at birth, mean } \pm \text { SD } \\
\text { (range), week }\end{array}$ & $\begin{array}{l}33.5 \pm 3.50 \\
(26.1-38.3)\end{array}$ & $\begin{array}{l}32.9 \pm 4.49 \\
(27.3-40.1)\end{array}$ & $\begin{array}{l}36.3 \pm 2.84^{\mathrm{a}} \\
(28.4-40.6)\end{array}$ & $\begin{array}{l}36.3 \pm 2.74 \\
(29.3-41.3)\end{array}$ \\
\hline Weight, mean \pm SD (range), g & $\begin{array}{l}2121.2 \pm 681.31 \\
(692-2938)\end{array}$ & $\begin{array}{l}2015.0 \pm 805.67 \\
(1010-3330)\end{array}$ & $\begin{array}{l}2761.9 \pm 567.84^{\mathrm{a}} \\
(1280-3820)\end{array}$ & $\begin{array}{l}2844.4 \pm 664.80^{\mathrm{b}} \\
(1280-4230)\end{array}$ \\
\hline $\begin{array}{l}\text { Head circumference, } \\
\text { mean } \pm \mathrm{SD} \text { (range), cm }\end{array}$ & $\begin{array}{l}30.1 \pm 3.06 \\
(23.0-33.3)\end{array}$ & $\begin{array}{l}29.6 \pm 2.79 \\
(24.5-34.5)\end{array}$ & $\begin{array}{l}33.0 \pm 2.18^{\mathrm{C}} \\
(28.0-36.0)\end{array}$ & $\begin{array}{l}33.0 \pm 1.89^{d} \\
(28.3-35.4)\end{array}$ \\
\hline $\begin{array}{l}\text { Apgar score } 1 \text { minute after } \\
\text { birth, median (range) }\end{array}$ & $\begin{array}{l}8 \\
(3-9)\end{array}$ & $\begin{array}{l}8 \\
(3-9)\end{array}$ & $\begin{array}{l}9^{\mathrm{a}} \\
(3-10)\end{array}$ & $\begin{array}{l}9 \\
(4-10)\end{array}$ \\
\hline $\begin{array}{l}\text { Apgar score } 5 \text { minutes after } \\
\text { birth, median (range) }\end{array}$ & $\begin{array}{l}9 \\
(7-10)\end{array}$ & $\begin{array}{l}9 \\
(6-10)\end{array}$ & $\begin{array}{l}9^{\mathrm{a}} \\
(6-10)\end{array}$ & $\begin{array}{l}9 \\
(7-10)\end{array}$ \\
\hline
\end{tabular}

${ }^{a} n=46 ;{ }^{b} n=49 ;{ }^{c} n=43 ;{ }^{d} n=42$

$G A$, gestational age; $S D$, standard deviation 